\title{
QUALIDADE DO SÊMEN DE TOUROS DAS RAÇAS ABERDEEN ANGUS E BRANGUS-IBAGÉ EM FRENTE À DEGENERAÇÃO TESTICULAR EXPERIMENTAL INDUZIDA POR DEXAMETASONA
}

\author{
BULLS SEMEN QUALITY OF ABERDEEN ANGUS AND BRANGUS-IBAGÉ BREEDS AFTER \\ EXPERIMENTAL TESTICULAR DEGENERATION INDUCED BY DEXAMETHASONE
}

\author{
Marilise Mesquita Horn ${ }^{1}$ José Carlos Ferrugem Moraes ${ }^{2}$ Carlos Salvador Galina $^{3}$
}

RESUMO

Foi administrada dexametasona para indução de degeneração testicular experimental em touros de uma raça taurina pura (Aberdeen Angus) e de sua sintética derivada (Brangus-Ibagé). O objetivo deste estudo foi o de averiguar a diferença de sensibilidade na função gametogênica em frente à degeneração experimental. Para tal, foram avaliados os aspectos físicos e morfológicos do sêmen. Os dados obtidos foram analisados considerando o dia de coleta e genótipo. Os resultados revelaram que os dois grupos raciais comportaram-se semelhantemente ao longo das coletas. A despeito do número de animais empregados, o uso da degeneração testicular experimental possibilitou evidenciar que não há diferença entre as duas raças quanto à intensidade da degeneração e tempo necessário para o restabelecimento do quadro espermático normal.

Palavras-chave: qualidade de sêmen, degeneração testicular, dexametasona, touros, raças.

\section{SUMMARY}

Testicular degeneration was induced by dexamethasone injection in bulls from a European breed Aberdeen Angus and a derived synthetic crossbreed (BrangusIbagé). Aiming to investigate differential sensibility on gametogenic function in these genotypes, physical and morphological semen characteristics were evaluated. The data analysed considered the day of the semen samples and the breed. Our results reveal that both breeds behave similarly during the experimental period. Even considering the number of bulls used in this experiment, the employed methodology gives no evidence of any difference between breeds, intensity of degeneration or time required to the reestablishment of normal sperm frequencies after experimental induction of testicular degeneration.
Key words: semen quality, testicular degeneration, dexamethasone, bulls, breeds.

\section{INTRODUÇÃO}

A variabilidade constatada na qualidade do sêmen de touros está intimamente correlacionada com alterações ambientais de diferentes origens. GALINA \& ARTHUR (1991), em uma revisão sobre aspectos reprodutivos em touros nos trópicos, salientam a importância da variação sazonal sobre a concentração espermática e percentagem de espermatozóides com defeitos morfológicos, indicando que baixa qualidade de sêmen em alguns animais pode ser devido ao desconforto proporcionado pelas condições ambientais desfavoráveis. SKINNER \& LOUW (1966) utilizaram touros jovens Bos taurus e Bos indicus para estudar a espermatogênese em temperaturas ambientais altas e buscaram averiguar o local e a natureza do dano no ciclo espermatogênico. As características espermáticas dos touros Bos indicus foram menos afetadas que a dos touros Bos taurus, em função da maior adaptabilidade dos indianos a altas temperaturas. Da mesma maneira, SILVA et al. (1991) observaram maiores efeitos deletérios na qualidade seminal decorrentes de baixa disponibilidade alimentar, precipitação pluvial e temperatura ambiental em touros cruzas Nelore com

\footnotetext{
${ }^{1}$ Aluno de mestrado do Curso de Pós-graduação em Ciências Veterinárias, Faculdade de Veterinária, Universidade Federal do Rio Grande do Sul, Porto Alegre, RS.

${ }^{2}$ Centro de Pesquisa de Pecuária dos Campos Sulbrasileiros, Empresa Brasileira de Pesquisa Agropecuária, 96400-970, Bagé, RS, email: ferrugem@cppsul.embrapa.br, Autor para correspondência.

${ }^{3}$ Faculdad de Veterinaria, Universidad Autonoma de México, AP 22060, D.F 14000, México.

Recebido para publicação em 24.07.98. Aprovado em 04.11.98
} 
Fleckvieh e Chianina do que em touros Nelore puro sob condições subtropicais. Sob as condições de clima temperado, do sul do Rio Grande do Sul, não seriam esperadas diferenças na espermatogênese entre estes grupos genéticos em função de altas temperaturas ambientais. No entanto, existem algumas indicações de que touros de raças sintéticas mostram maior percentagem de descarte por alterações seminais do que touros de raças puras européias criados nos mesmos sistemas de criação (MORAES et al., 1998).

As hipóteses testadas no presente estudo foram de que touros de genótipos sintéticos, oriundos de cruzamentos de Bos indicus com Bos taurus, são mais suscetíveis à degeneração testicular do que touros de genótipos puros europeus e que os mecanismos responsáveis pela regeneração testicular e/ou seleção gamética não funcionam com a mesma eficiência nos dois grupos genéticos, o que poderia explicar a maior freqüência de diagnósticos de degeneração testicular nos genótipos sintéticos.

O objetivo foi averiguar o comprometimento da espermatogênese e o tempo de recuperação após uma degeneração testicular experimental em touros contemporâneos de raça européia pura e de genótipos sintéticos (oriundos de cruzamentos entre Bos taurus e Bos indicus).

\section{MATERIAL E MÉTODOS}

Foram utilizados quatro touros da raça Aberdeen Angus, representando o genótipo puro e três touros da raça Brangus-Ibagé (5/8 A Angus + 3/8 Nelore), representando o genótipo sintético. O experimento foi conduzido nas dependências do Centro de Pesquisa Pecuária dos Campos Sulbrasileiros (CPPSUL)/Embrapa, Bagé, RS. Os animais estavam com dois anos de idade, sem experiência sexual, criados em campo nativo, semelhantes quanto à condição corporal, que foi classificada como quatro, numa escala de 1-5. Os animais foram suplementados com silagem de sorgo, durante o período experimental. $\mathrm{O}$ tratamento consistiu na aplicação de $20 \mathrm{mg}$ de dexametasona $/$ dia por sete dias consecutivos em todos os touros, independentemente de peso (BARTH,1993), objetivando induzir um quadro de degeneração testicular leve, para estudo do quadro espermático nos dois grupos.

O sêmen destes animais foi coletado através de eletroejaculação duas vezes por semana. As coletas tiveram início duas semanas antes do início do tratamento (27/01/97) e desenvolveram-se até nove semanas após, num total de 21 , em cada touro. Cada coleta está representada pelo dia referente ao início do tratamento (dia zero): primeira coleta dia -
10 , segunda -7 e assim sucessivamente até a vigésima primeira coleta, 58 dias do início do tratamento.

Os componentes da avaliação andrológica medidos foram: aspecto do ejaculado (ASP), avaliado macroscopicamente com a finalidade de estimar a concentração de espermatozóides no ejaculado, em uma escala de 1-4, onde 1 representou o aspecto cremoso, 2 leitoso, 3 opalescente e 4 aquoso; turbilhonamento (TUR), avaliado em sêmen puro, em aumento de 40x numa escala de 1 (sem turbilhonamento) a 5 (máximo); motilidade espermática (MOT) em \% e vigor (VIG) em uma escala de 1-5 aferidos entre lâmina e lamínula em aumento de 100x e 400x; morfologia espermática avaliada em lâmina corada (CEROVSKY, 1976) em um aumento de 1000x num total de 200 células por amostra, considerando $\%$ de espermatozóides normais (NOR); \% de defeitos de cabeça (CAB); \% de defeitos de peça intermediária (PI), incluindo gota citoplasmática proximal e distal; $\%$ de defeitos de cauda (CAU); \% de defeitos de acrossoma (ACR) e \% de cabeça solta normal (CSN).

As variáveis mensuradas foram submetidas à análise de variância considerando os fatores: genótipo, touro dentro de genótipo e dia da coleta de sêmen e interações de primeira ordem. Estas variáveis foram transformadas em "ranks", visando à obtenção de distribuição normal dos dados.

\section{RESULTADOS E DISCUSSÃO}

Todos os indicadores da qualidade do sêmen variaram significativamente $(\mathrm{P}<0,05)$ ao longo dos dias de coleta. Esses dados indicam a propriedade do modelo testado como agente causal de uma degeneração testicular. As médias das variáveis ASP, TUR e VIG apresentaram decréscimo qualitativo significativo entre o $9^{\circ}$ e $40^{\circ}$ dia do início das injeções de dexametasona. Na figura 1, é ilustrada a intensidade e o período de duração da degeneração nos animais tratados, através da motilidade espermática e \% de espermatozóides normais. As médias de MOT e NOR apresentaram decréscimo já no segundo dia após o início do tratamento. A MOT apresentou decréscimo de $70 \%$ no dia -1 para $20 \%$ no dia 19. A NOR passou de $89 \%$ no dia -1 para $51 \%$ no dia 23 e os valores iniciais foram novamente atingidos a partir do $44^{\circ}$ dia do início do tratamento.

$\mathrm{O}$ quadro de degeneração testicular provocado pela dexametasona e o retorno ao quadro espermático basal em menos de 58 dias reforçam a validade do modelo proposto por COULTER (1976) e BARTH (1993) para outros estudos referentes à espermatogênese dos bovinos. A detecção de alterações em todos os componentes da avaliação andro- 


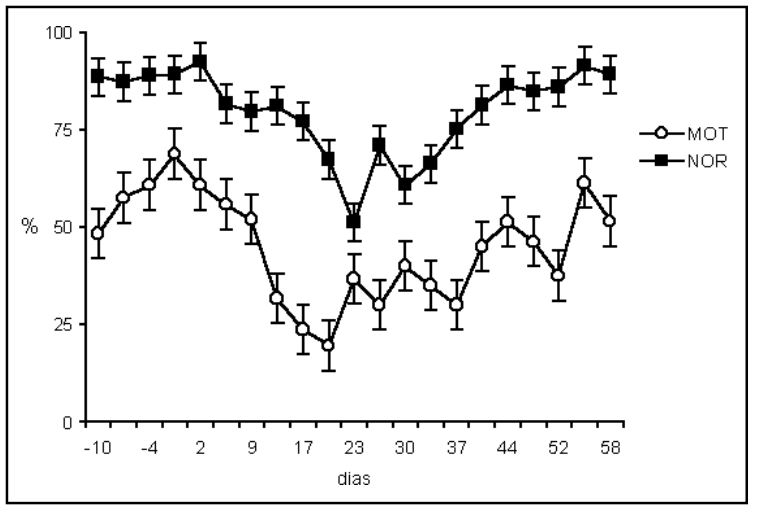

Figura 1 - Variação no percentual de motilidade espermática (MOT) e de células normais (NOR) ao longo do período experimental nos touros tratados.

lógica evidencia que a aplicação de dexametasona influenciou de uma maneira direta ou indireta a função espermática. $\mathrm{O}$ mecanismo pelo qual a dexametasona causa distúrbio na espermatogênese ainda não está totalmente esclarecido. THIBIER \& ROLAND (1976) postularam efeito indireto pela redução da liberação de LH e conseqüentemente de testosterona. WELSH et al. (1979) observaram que os aumentos dos níveis sangüíneos de cortisol freqüentemente coincidiam com os níveis basais de $\mathrm{LH}$ e testosterona, relação não encontrada em humanos por DOERR \& PIRKE (1976), que evidenciaram inibição direta da biossíntese de testosterona pelas altas taxas plasmáticas de cortisol ou dexametasona. Reiterando essa possibilidade, BAMBINO \& HSUEH (1981) demonstraram o efeito inibitório dos glucocorticóides sintéticos e naturais nos receptores de LH nas células de Leydig e na esteroidogênese. Neste estudo, não foram efetuadas dosagens de testosterona e LH que poderiam contribuir tanto no estudo do mecanismo de ação da dexametasona quanto na verificação das hipóteses testadas.

As médias de todas as variáveis medidas estão apresentadas por genótipo na tabela 1. A tendência esperada de menor qualidade de sêmen no genótipo sintético não foi confirmada, sendo observado o contrário em inúmeros indicadores. No caso específico dos defeitos de acrossoma, foi detectada uma maior freqüência nos touros sintéticos, porém, sua magnitude não indica uma alteração grave na espermiogênese. Já os defeitos de cauda (CAU) foram constatados com média superior nos touros puros e com apresentação peculiar entre as duas raças ao longo dos dias de coleta (interação genótipo e coletas, $\mathrm{P}<0,05)$. A hipótese testada de que seria possível através de avaliações repetidas identificar diferenças entre os genótipos, não se confirmou, em função da alta variação individual, indicando que novos estudos nessa área devem incluir maior número de indivíduos e apenas alguns indicadores da qualidade seminal.

Os resultados permitem inferir que os genótipos são semelhantes e que os sintéticos não são identificados por mais tempo em degeneração testicular, portanto, refutando as hipóteses testadas. No entanto, algumas peculiaridades foram identificadas com respeito a defeitos espermáticos específicos (CAU e CSN), caracterizadas por interações significativas entre genótipo e dias de coleta $(\mathrm{P}<0,05)$.

$\mathrm{Na}$ figura 2, são apresentadas as médias observadas para CAU e CSN nos dois genótipos. O genótipo puro apresentou uma elevação nos defeitos de cauda do $5^{\circ}$ ao $37^{\circ}$ dia após início do tratamento, o que não ocorreu com o sintético, que manteve os mesmos índices de defeitos de cauda durante todo o período de avaliações, com um pequeno aumento entre as coletas do dia 17 ao 23. Já para CSN, o genótipo puro apresentou as maiores taxas de cabeça solta normal entre o $17^{\circ}$ e $25^{\circ}$ dia e o sintético entre o $23^{\circ}$ e $40^{\circ}$ dias, demonstrando que o puro apresentou mais precocemente esta anormalidade. As maiores taxas de CSN foram constatadas no genótipo puro entre o $17^{\circ}$ e $25^{\circ}$ dia e no sintético entre o $23^{\circ}$ e $40^{\circ}$ dia de coleta. Não foi objetivo verificar o dia exato do início do aparecimento das anormalidades espermáticas nos dois genótipos, no entanto, consi-

Tabela 1 - Médias ajustadas das variáveis medidas nos dois genótipos de touros.

\begin{tabular}{crr}
\hline Variáveis & Genótipo puro & Genótipo sintético \\
\hline Aspecto do ejaculado (1-4) & $1,61 \pm 0,14$ & $1,92 \pm 0,17$ \\
Turbilhonamento (1-5) & $1,43 \pm 0,28$ & $1,42 \pm 0,17$ \\
Motilidade (\%) & $41,51 \pm 4,47$ & $48,45 \pm 5,50$ \\
Vigor (1-5) & $2,42 \pm 0,11$ & $3,10 \pm 0,14$ \\
Perímetro escrotal (cm) & $33,34 \pm 0,58$ & $31,58 \pm 0,74$ \\
Espermatozóides normais (\%) & $73,08 \pm 2,03$ & $86,53 \pm 2,49$ \\
Defeitos de acrossoma (\%)* & $1,33 \pm 0,28$ & $2,04 \pm 0,34$ \\
Defeitos de cabeça (\%) & $2,77 \pm 0,31$ & $1,94 \pm 0,37$ \\
Defeitos de peça intermediária. (\%) & $5,53 \pm 1,23$ & $2,96 \pm 1,51$ \\
Defeitos de cauda (\%)* & $14,14 \pm 2,33$ & $3,09 \pm 2,86$ \\
Cabeça solta normal (\%) & $3,26 \pm 0,57$ & $3,49 \pm 0,69$
\end{tabular}

* diferem na linha $\mathrm{P}<0.05$.

Ciência Rural, v. 29, n. 3, 1999. 


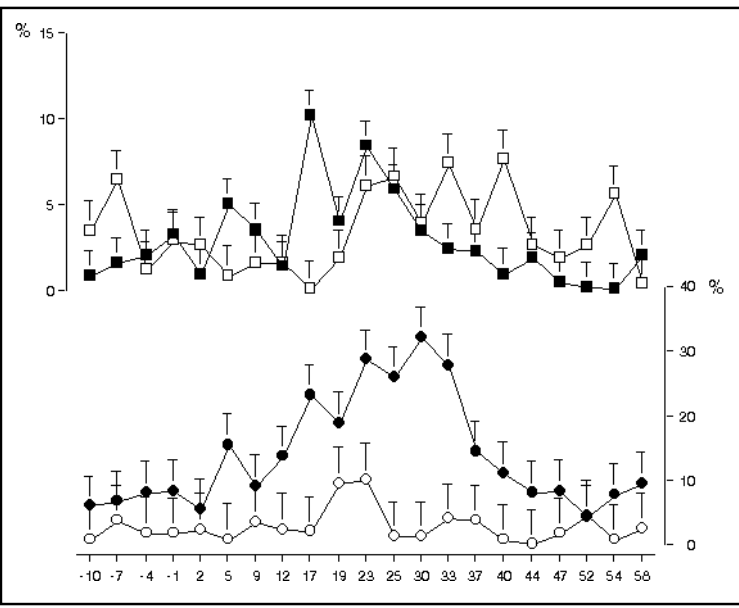

Figura 2 - Variação no percentual de defeitos de cauda (Genótipo puro e sintético $\mathbf{0}$ ) e cabeça solta normal (genótipo puro $\square$ e sintético $\square$ ) ao longo do período experimental.

derando o momento da detecção das anormalidades, pode-se inferir que os danos devem ter ocorrido no testículo.

\section{CONCLUSÃO}

De acordo com os resultados obtidos, é possível concluir que a aplicação de dexametasona resulta numa degeneração testicular leve, com alterações de todas as características mensuradas no sêmen dos animais tratados, comprometendo a espermatogênese e o tempo de recuperação por um período semelhante nos dois genótipos testados. Por outro lado, a observação de alguns defeitos espermáticos, em momentos distintos, indicam peculiaridades na espermatogênese dos genótipos puros e sintéticos, que devem ser melhor investigadas através de estudos que identifiquem a origem e local de reabsorção/seleção destes defeitos ao longo do epitélio seminífero e dutos excretores.

\section{FONTES DE AQUISIÇÃO}

a - Azium, Schering

\section{REFERÊNCIAS BIBLIOGRÁFICAS}

BAMBINO, T.H., HSUEH, A.J. Direct inhibitory effect of glucocorticoids upon testicular luteinizing hormone receptor and steroidogenesis in vivo and in vitro. Endocrinology, v.108, p. 2142-2148, 1981

BARTH, A.D. Insights to the pathogenesis of sperm abnormalities in bulls. Rev Bras Reprod Anim, v. 1, p. 1-14, 1993.

CEROVSKY, J.A. A new staining procedure for boar spermatozoa. Zivocisna Vyroba, v. 21, p. 351-362, 1976.

COULTER, G.H. Effect of dexamethasone on the incidence of the crater defect of bovine sperm. In: INTERNATIONAL CONGRESS ON ANIMAL REPRODUCTION AND ARTIFICIAL INSEMINATION, 8, Cracow. Proceedings... p. 694-697, 1976.

DOERR, P., PIRKE, K.M. Cortisol-induced suppression of plasma testosterone in normal adult males. J Clin End Metab v. 43 , p. $623-629,1976$.

GALINA, C.S., ARTHUR, G.H. Review of cattle reproduction in the tropics. Part 6. The male. Anim Breed Abs, v. 59, p. 403412,1991

MORAES, J.C.F., HORN, M.M., ROSADO, A. Exame andrológico em touros: qualidade dos indicadores da aptidão reprodutiva em distintos grupos raciais. Ciência Rural, v. 28, n. 4 , p. $647-652,1998$

SILVA, A.E.D.F., DODE, M.A., PORTO, J.C.A. Estacionalidade na atividade sexual de machos bovinos Nelore e mestiços Fleckvieh x Nelore e Chianina x Nelore: características espermáticas. Pesq Agropec Bras, v. 26, p. 1784-1790, 1991.

SKINNER, J.D., LOUW, G.N. Heat stress and spermatogenesis in Bos indicus and Bos taurus cattle. J Appl Phys, v. 21, p. 1784-1790, 1966.

THIBIER, M., ROLAND, O. The effect of dexamethasone (dmx) on circulating testosterone $(\mathrm{t})$ and luteinizing hormone $(\mathrm{lh})$ in young postpubertal bulls. Theriogenology, v. 5, p. 53-60, 1976.

WELSH, T.H, McCRAW, R.L, JOHNSON, B.H. Influence of corticosteroids on testosterone production in the bull. Biol Reprod, v. 21, p. 755-763, 1979. 